\title{
COMUNICAÇÕES
}

Defesas de Teses de Doutorado e Dissertações de Mestrado do Curso de Pós-Graduação em Controladoria e Contabilidade da FEA-USP

\section{DISSERTAÇÕES DE MESTRADO}

“A EVIDENCIAÇÃO DAS INFORMAÇÕES TRIBUTÁRIAS PELAS INSTITUIÇÕES FINANCEIRAS EM FACE DA CONVERGÊNCIA PARA AS NORMAS INTERNACIONAIS"

\section{TATIANA LOPES}

Orientador: Prof. Dr. Iran Siqueira Lima

Data de defesa: 15.08.2008

"O IMPACTO DA PRIMEIRA ADOÇÃO DAS NORMAS IFRS NAS EMPRESAS EUROPÉIAS QUÍMICAS E DE MINERAÇÃO: PESQUISA DESCRITIVA"

\section{MARCELO CAMBRIA}

Orientador: Prof. Dr. Luiz Nelson Guedes de Carvalho

Data de defesa: 20.08.2008

"LUCRO REAL OU LUCRO: POR QUAL OPTAR?"

\section{CARMINE RULLO}

Orientador: Prof. Dr. Ariovaldo dos Santos

Data de defesa: 03.09.2008
"ANÁLISE DAS CARACTERÍSTICAS DOS SISTEMAS DE SEGURO DE DEPÓSITO: BRASIL E ALGUMAS PRÁTICAS INTERNACIONAIS"

\section{LUÍS EDUARDO MIZU DA SILVA}

Orientador: Prof. Dr. Iran Siqueira Lima

Data de defesa: 05.09.2008

"ESTIMATIVA DO PRÊMIO PELO RISCO PAÍS COM A APLICAÇÃO DO MODELO AEG"

\section{GUILHERME GARCIA BELLOQUE}

Orientador: Prof. Dr. Alexsandro Broedel Lopes

Data de defesa: 01.10.2008

\section{ERRATA}

No artigo “Índices de Risco Sistêmico para o Setor Bancário" publicado no volume 19, edição 47, maio/agosto de 2008, por um lapso não constaram as notas de rodapé 1 e 2 abaixo:

1 A tabela de distribuição normal padronizada está disponível em Downing e Clark (1998, p. 448).

2 Acrônimo de Capital, Assets, Management, Earnings, Liquidity e Sensibility, que expressa a metodologia de avaliação de instituições financeiras desenvolvida pelos supervisores bancários norte-americanos. 